On the 3 rd of September at about a quarter before one p. $m$. I set the Equatoreal to the position of the comet deduced from the epheneris published in No. 869 of the Astr. Nachr., and on applying my eye to the telescope 1 immediately detected the comet almost in the centre of the field. Its appearance was planetary, the nucleus was round, well defined, of a whitish colour, and about 9" diameter. I could not detect the slightest appearance of a tail. The power used was 92 , but with the view of obtaining the position of the comet more accurately I put on the parallel wire-micrometer (power 132) with which it was well seen, but before I could complete my arrangements for an observation clouds came over and 1 did not see it again till $2 \mathrm{p}$. m. Between 2 and 4 p. m. I saw the comet again two or three times for very short intervals, but it was exceedingly faint, and clouds and haze prevented further observation.

P h, c a e a.

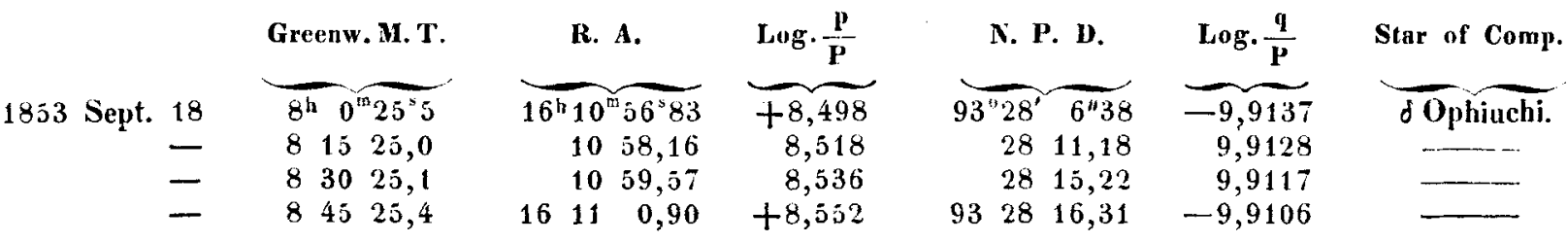

The observations are corrected for refraction.

The following is the assumed mean place of the star of comparison for 1853,0 .

$$
\delta \text { Ophiuchi } \quad \frac{\text { R. A. }}{16^{\mathrm{n}} 6^{\mathrm{n}} 38^{\mathrm{*}} 73} \quad \frac{\text { N. P. D. }}{93^{\circ} 18^{7} 42^{\prime \prime} \mathbf{j} 6} \quad \underbrace{\text { Authority. }}_{\text {Greenwich Observations 1849-50. }}
$$

\begin{tabular}{|c|c|c|c|c|c|c|}
\hline \multirow{5}{*}{1853} & Greenw. M. T. & R. A. & Log. $\frac{p}{P}$ & s. P. D. & Log. $\frac{q}{p}$ & Stitrs of Comp \\
\hline & $\underbrace{}_{10^{\mathrm{h}} 43^{\mathrm{m}} 29^{\mathrm{s}} 2}$ & $23^{\mathrm{h}} 12^{\mathrm{m}} 33^{\mathrm{s}} 17$ & $-7,910$ & $102^{\prime \prime} 13^{\prime} 11^{\prime \prime} 7^{\prime \prime}$ & $-9,9566$ & $\widehat{\text { B.A.C. } 8175}$ \\
\hline & $10 \quad 58 \quad 26,7$ & 1232,75 & $-7,740$ & 1313,0 & 9,9573 & \\
\hline & $\begin{array}{lll}11 & 13 & 23,9\end{array}$ & 1231,69 & $-7,454$ & 1316,9 & 9,9577 & 8199 \\
\hline & 113320,1 & $\begin{array}{lll}23 & 12 & 30,70\end{array}$ & $+6,852$ & $\begin{array}{lll}102 & 13 & 18,6\end{array}$ & $-9,9578$ & 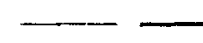 \\
\hline
\end{tabular}

T he t is.

The olservations are currected for refraction.

The following are the assumed mean places of the stars of comparison for 1853,0 .

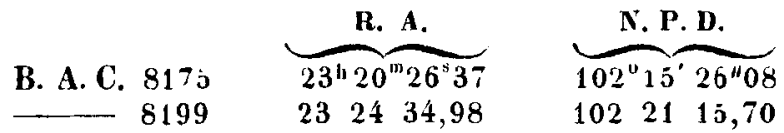

Liverpool Observatory 1853, Sept. 22.
British Association Catalogue.

John Hartnup.

\title{
Cometenbeobachtungen auf der Sternwarte zu Olmütz.
}

Die Beobachtungen des von Herrn Klinkerfues entdeckten Cometen sind an der hiesigen Sternwarte von der Witterung im Ganzen sehr begünstigt worden. Ich habe sie nicht ausschliesslich auf die Ortshestimmungen bezogen, sondern diesmal den physischen Erscheinungen eine hesondere Aufmerksamkeit zugewandt. Wegen der erheblichen Anzahl aller Beobachtungen sehe ich mich indessen genöthigt, die Mitthei- lungen darüber für die Astron. Nachrichteln, in 3 Abtheilungen zu geben. Der erste hier folgende Bericht enthält die sämmt. lichen Messungen an den Kreismikrumetern zweier Refractoren, alle in der Nacht oder in der Abenddämmerung ausgeführt. Der zweite Bericht wird die im vollen Tageslichte, Morgens, Mittags und Nachmittags, zwischen Aug. 30. $3^{\text {th }}$ und Sept. $4.5^{\text {h }}$ angestellten Cometenlieobachtungen bringen, deren 
nicht geringe $Z a h l$ mïglichst scharf zu berechnen, mich gegenwärtig noch beschäftigt. In einem $3^{+e n}$ Aufsatze werde ich noch später nittheileu: Beobachtungen über die sämmtlichen auf Licht, Farlie und Diniensionen des Cometen bexüglicben Erscheinungen, Mikrometermessungen des Kerns, Grösse und vermuthete selbstständige Bewegungen des Schweifes, so wie einige Cntersuchungen äber die Polarisation des Cometenlichts Vou allen diesen Dingen schweige ich also noch, und gehe sogleich nur Mittheihng der Kreismikrometer-beobachtungen über, welche alle, mit Ausnahme einer von Hru. v. Unkrechtsberg gemachtell, von nir allein angestellt worden sind. Von der Entreckung des Cometen crhielt ich, von einer Reise zurückgekehrt, erst am $24^{\text {sten }}$ Juni durch die Astron. Nachr. Kunde. Die ersten Beobachtungen gelangen am $26^{\text {sten }}$ Juni; sie wurden dann zwischen Juli 13 und Juli 25 abermals durch eine andere Reise unterbrochen. Von Juli 25 setzte ich sie fort, bis nach Aug. 26 in der Abenddämmerung kein Vergleich. stern melır in der Nähe des Cometen gesehen werden konnte. Da der Comet seit dem Aufange Augusts sich dem Westhorizonte so stark näherte, dass er auf der Sternwarte nur $\frac{1}{4}$ Stunde lang gesehen werden konnte, liess Hr, v. Unkrechts. berg den kleinen. 3 fuss. Plössl'schen und parallaktischen Refractor in das benachbarte Seminar hringen, wo derselhe es aufstellte und sehr nabe orientirte. An diesem Orte, wo man den Comseten bis zu seinem Untergange an Horizonte des Sudetengehirges vafolgen komote, habe ich noch 5 Posi. tionen, und in Ganzen 50 erbalten. Nlle Beobachtungen sind völlig scharf relucirt; in Fällen, da die Zenithdistanz $78^{\circ}$ uberstieg, ist die Constante der Refraction wegen des Baro. meter- und Thermometerstandes verbessert worden.

In der folgenden Uebersicht ist die Beobachtung des Herm Prälaten durch ein $(t)$ bezeichnet; die am 3 fussigen Refractor erhaltenen Positionen sind von den übrigen durch ein (*) unterschieden.

Alle Zeiten sind mittlere Zeiten zu Olmütz.

$$
\begin{aligned}
& \varphi=49^{\circ} 35^{\prime} 40^{\prime \prime} \\
& l=15^{\mathrm{m}} 24^{\mathrm{s}} 6 \text { Ost von Berliu. }
\end{aligned}
$$

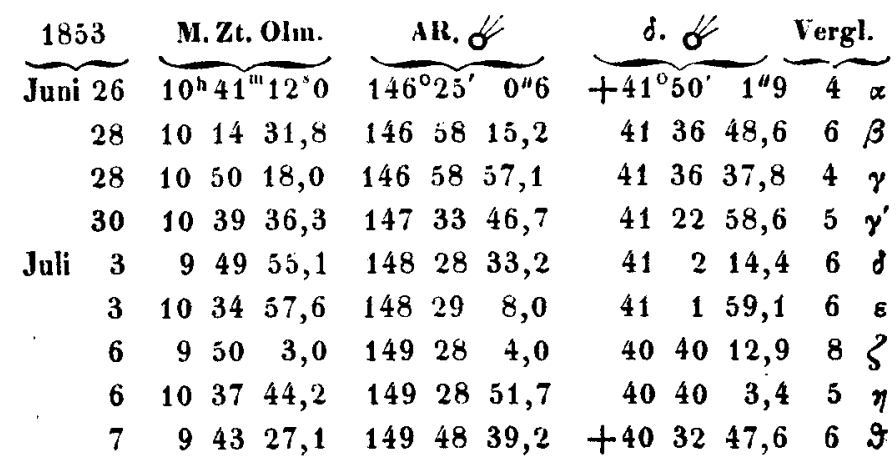

$18 \mathbf{3 3}$

$\overbrace{\text { Juli } 7}$

$$
\begin{aligned}
& 7 \\
& 9
\end{aligned}
$$

9

$$
9
$$$$
9
$$$$
12
$$$$
13
$$$$
25
$$$$
25
$$$$
26
$$$$
26
$$$$
27
$$$$
27
$$$$
28
$$$$
30
$$$$
31
$$

Aug. 1

$$
2
$$$$
\text { (*) } 3
$$

(*) 3$$
5
$$$$
9
$$$$
12
$$$$
13
$$$$
14
$$

(t) 1

15

16

$$
16
$$$$
19
$$$$
20
$$$$
22
$$

(*) 2

$\begin{array}{lllllll}22 & 8 & 40 & 6,6 & 172 & 47 & 40,0\end{array}$

$\begin{array}{llllllll}23 & 8 & 0 & 4,3 & 173 & 11 & 21,6\end{array}$

(*) $23 \quad 8 \quad 41 \quad 37,1 \quad 173 \quad 11 \quad 58,0$

$24 \div 47 \quad 15,9 \quad 173 \quad 3153,1$

(*) $24 \quad 8 \quad 36 \quad 13,2 \quad 173 \quad 32 \quad 24,5$

$\begin{array}{lllllll}25 & 7 & 43 & 21,2 & 173 & 47 & 23,9\end{array}$

(*) $25 \quad 8 \quad 27 \quad 54,8 \quad 173 \quad 47 \quad 40,4$

$\begin{array}{lllllll}26 & 7 & 41 & 9,3 & 173 & 57 & 0,9\end{array}$

1) Filarnikrometer. $\quad$ 2) Unsicher.

\section{Angenommene scheinbare Oerter der Vergleichsterne.}

Bis jetzt konnte ich nur Lal., Bess. Z., Piazai, den GeneralCatalogue und Rümker benutzen. Manche der hellern Sterne sind gewiss scharf bestinnt, doch standen mir andere Hülfs. mittel nicht zu Gebote. Je nach den Umständen habe ich 
die Gewichte für die verschiedenen Sterne angenommen, wobei einige Willkür nicht zu vermeiden ist. Ich beschränke mich hier ouf die Mittheilung der Sternörter, so wie ich sie zur Reduction der Beobachtungen gebraucht habe.

\begin{tabular}{|c|c|c|c|c|}
\hline & & $43^{m} 8^{k} 28$ & $+41^{\circ} 39^{\prime} 31^{\prime \prime} 6$ & B. Z. 454, Lal. 19283 \\
\hline 0 & 9 & 4826,29 & 415336,5 & -454 \\
\hline & 9 & $48 \quad 39,95$ & $\begin{array}{lll}41 & 45 & 19,8\end{array}$ & $\longrightarrow 454$, Lal. 19430 \\
\hline & 9 & $48 \quad 39,94$ & $41 \quad 45 \quad 19,7$ & - \\
\hline & 9 & $54 \quad 41,39$ & 412110,0 & Am Kreismikr. bestimmt. \\
\hline & 9 & 5534,49 & $41 \quad 22 \quad 33,7$ & B. Z. 454 \\
\hline & 9 & $58 \quad 13,40$ & $40 \quad 40 \quad 3,3$ & Am Kreismikr. bestimnt. \\
\hline & 9 & 5739,86 & $40 \quad 13 \quad 11,8$ & $\begin{array}{r}\text { B. Z. } 450,498, \text { Lal. } 1968 \\
1968\end{array}$ \\
\hline & 9 & $57 \quad 3,58$ & 401750,2 & $\begin{array}{r}\text { Lal. } 1966 \\
1966\end{array}$ \\
\hline & 9 & 5651,58 & $4035 \quad 0,4$ & Am Kreismikr. bestimmt. \\
\hline & 9 & 5739,86 & $40 \quad 13 \quad 11,7$ & $=\eta$. \\
\hline & 10 & 20,66 & $40 \quad 5 \quad 42,0$ & B. Z. 450,498 \\
\hline & 10 & 151,19 & $4038 \quad 14,4$ & Am Kreismikr. bestimnıt. \\
\hline$\mu$ & 10 & 8,3 & 3953 & Unbekannt. \\
\hline & 10 & 543,93 & $\begin{array}{lll}40 & 9 & 2,1\end{array}$ & B. Z. 498 . \\
\hline & 10 & 8,6 & 402 & Unbekannt. \\
\hline & 10 & 818,54 & $\begin{array}{lll}39 & 15 & 9, \mathbf{j}\end{array}$ & B. Z. 498 \\
\hline$\pi$ & 10 & 2939,11 & $\begin{array}{lll}37 & 13 & 17,2\end{array}$ & -407 \\
\hline 8 & 10 & $28 \quad 14,29$ & $38 \quad 6 \quad 34,8$ & -407 \\
\hline D & 10 & 2754,18 & $37 \quad 5 \quad 23,7$ & $-407,499$ \\
\hline T & 10 & $31 \quad 12,53$ & 371712,8 & -407 \\
\hline$\varphi$ & 10 & $34 \quad 1,95$ & 371719,7 & -407 \\
\hline$x$ & 10 & $33 \quad 6,89$ & $37 \quad 50 \quad 24,4$ & -407 \\
\hline$\varphi^{\prime}$ & 10 & $34 \quad 1,94$ & $\begin{array}{lll}37 & 17 & 19,7\end{array}$ & $=\varnothing$ \\
\hline$\psi$ & 10 & 4150,39 & $\begin{array}{lll}36 & 52 & 3 j, 7\end{array}$ & B. Z. 411,499 \\
\hline 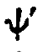 & 10 & 4150,38 & $36 \quad j 2 \quad 35,6$ & $=\psi$ \\
\hline$\psi^{\prime \prime}$ & 10 & 4150,37 & $3652 \quad 35,4$ & $=\psi$ \\
\hline & 10 & 4251,30 & $\begin{array}{lll}36 & 37 & 8,5\end{array}$ & B. Z. 491,499 \\
\hline
\end{tabular}

In 499 ist $\delta$ un $1 \frac{1}{2}$ kleiner. Diese habe ich angenommen.
$\begin{array}{lllll}\text { a } & 10 & 48 & 55,29\end{array}$
$35 \quad 58 \quad 59,9$ Lal. 21025
$\begin{array}{llll}b & 10 & 49 & 37,33\end{array}$
$\begin{array}{llll}35 & 24 & 47,4\end{array}$
B. Z. 491,499

Ich habe die Position (d) ans 499 angenommen.
c 11154,90
$\begin{array}{lll}33 & 19 & 41,8\end{array}$
B. Z. 357
d $\quad 1110 \quad 19,83$
$322124,1=\xi$ Ursae maj. = Rümk. Nr. 3509. Bei guter Luft kounte ich den Stern bei 40 mal. Vergrösserung doppelt erkennen.

$\begin{array}{rrrrrrrl}d^{\prime} & 11 & 10 & 19,82 & 32 & 21 & 23,9 & = \\ d^{\prime \prime} & 11 & 10 & 19,82 & 32 & 21 & 23,8 & =d \\ e & 11 & 17 & 48,11 & 30 & 47 & 42,5 & \text { B. Z. } 501, \text { Lal. } 21734 \\ e^{\prime} & 11 & 17 & 48,11 & 30 & 47 & 42,4 & = \\ f & 11 & 17 & 24,51 & 30 & 34 & 13,6 & \text { Lal. } 21718 \\ g & 11 & 28 & 33,09 & +28 & 35 & 41,1 & \text { Lal. } 22018\end{array}$

Piazzi 111. (XI.) Rïmker 3649

$$
\begin{aligned}
& g^{\prime} 11^{\mathrm{b}} 28^{\mathrm{n}} 33^{\mathrm{s}} 08+28^{\mathrm{n}} 35^{\prime} 40^{\prime \prime} 9=g \\
& \begin{array}{lllllllll}
h & 11 & 31 & 47,60 & 26 & 7 & 7,9 & \text { B. Z. } 500
\end{array}
\end{aligned}
$$

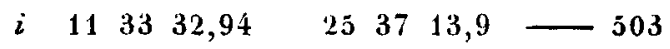

$$
\begin{aligned}
& \text { k } 113229,54 \quad 243121,0-353 \\
& \begin{array}{llllllll}
l & 11 & 33 & 8,10 & 22 & 10 & 11,4 & \text { Lal. } 22111
\end{array} \\
& \text { Piaza (XI.) B. Z. 453, 496. Rïmker } 3686 . \\
& \text { m } 113426,72 \quad 23 \quad 145,5 \text { Lal. } 22144 \text {. }
\end{aligned}
$$

B. Z. 496. Rïmker 3697 .

$\begin{array}{lllllll}n & 11 & 40 & 23,58 & +21 & 2 & 13,2\end{array}$. Lal. 22293

Piazzi XI., B. Z. 467, Rünker 3742 und Gen. Catal. Es ist $\varepsilon$ Ceonis. Die AlR. konnte ich nur unter Annahme eirnel eigenen Bewrgung von $-0^{s} 0118$ zur Jebereinstinmung luiugen.

$$
Z \text { u } \leq \text { a } t z \text {. }
$$

Zu folgenden Beolachtungen war der Vergleichstern nicht zu ermittelı.

$$
\begin{aligned}
& \text { AR. ơ o. ơ } \\
& \text { Juli } 129^{\mathrm{h} 55^{\mathrm{m}}} 5^{\mathrm{s}} 6 \text { AR. } \mu-1^{\mathrm{m}} 45^{\mathrm{s}} 86 \quad \delta \mu+0^{\prime} 41^{\prime \prime} 50 \quad 6
\end{aligned}
$$

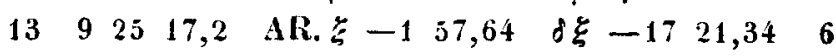

\section{Bemerkungen über die Sicherheit dieser Beobachtungen.}

Schon früher, hei Gelegenheit der Erscheinung des Encke. schen Cometen inı Jahre 1848, habe ich für meine Bonner Beobachtungen die mittleren und wahrscheinlichen Fehler der A hstïnde des Cometen run den Vergleichsternen mitgetheilt. Man findet diese Zahlen in den Astr. Nachr. Nr. 660 p. 183. Man erhält durch diese Werthe wenigstens ein Urtheil über die verhältnissnässige Sicherheit der Beobachtungen unter sich, und mit Ergehnissen ähulicher Art hei einem andern Cometen verglichen, Kenutniss von dem Einflusse, den das Licht und die Gestalt des Cometen, so wie seine Höhe äber dem Horizonle, auf die Genauigkeit der Messungen auszuüben vermochte. Bei diesen Cometen habe ich wiederum solche Rechnungen ausgeführt, awar keineswegs in aller Schäre, sondern nur in soweit, dass sie dem angedeuteten Zweck dienen. Ich hahe für jede Beohachtung eines Abends, mit der aus Bruhns Ephemeride annähernd bekannten Bewegung des Cometen, alle eimzelnen Messungen auf das Mitfel der Zeiten reducirt, aus den Abweichungen (R. - B.) die Quadratsummen gehildet, und die mittlern Fehler e in es Durchganges $=\varepsilon$, in den wahrscheinlichen Fehler des Mittels $=\varepsilon^{\prime \prime}$ berechnet. Ich beahsichtige also nur, die verhältliche Sicherheit meiner Beohachtungen unter sich hetrachtet, beilïufig darzustellea. Was wirklich der wahrscheinliche Fehler solcher Beobachtungen ist, wohei so viele Umstände Einfluss haben, wird erst erhellen, wenn sie durch eine definitive Bahn scharf verglichen worden sind. Man wird dann übersichtlich eine Tafel 
üher den wirklichen Werth der Beobachtungen verschiedener Steruwarten zusammenstellen, wie solche z. B. Sonntag in seiner Berechnung der Bahn des Petersen'schen Cometen von 1850 geliefert hat (A. N. Nr. 803 p. 17j).

In Betreff der folgender, Zahlen bemerke ich, dass ich nur die 2. Beub. vom $27^{\text {sten }}$ huli und die vom $5^{\text {ten }}$ August ausgeschlossen habe, weil mir die Ursachen bekannt sind, weshalb sie so mangelhatt ausfielen. Die Zahlen sollen also ausdrücken: den wahrscheinlichen Fehler dis Nittels aller, an einem Abende gemessenen Abstände des Cometen von den Vergleichsternen. Jeder uur cinmalige Durchgang ist nicht berücksichtigt.

Für den 5 fuss. Refractor.

\begin{tabular}{|c|c|c|c|}
\hline & $\varepsilon^{u}$ & AK. & $\varepsilon^{\prime \prime}$ iu $\delta$. \\
\hline Juni 26 & $\pm 0^{8} 116$ & $\cos 41^{\circ} 8$ & $\pm 3 " 81$ \\
\hline 28 & 0,067 & $\cos 41,6$ & 0,96 \\
\hline 28 & 0,081 & $\operatorname{ros} 41,6$ & 1,97 \\
\hline 30 & 0,165 & $\cos 41,4$ & 2,25 \\
\hline Juli & 0,068 & $\cos 41,0$ & 1,15 \\
\hline 3 & 0,053 & $\cos 41,0$ & 0,82 \\
\hline 6 & 0,041 & $\cos 40,7$ & 2,33 \\
\hline 6 & 0,076 & os 40,7 & 0,93 \\
\hline 7 & 0,062 & $\cos 40,6$ & 1,20 \\
\hline 7 & $\pm 0,067$ & $\operatorname{ros} 40,5$ & $\pm 1,79$ \\
\hline
\end{tabular}

\begin{tabular}{|c|c|c|}
\hline$e^{\prime \prime}$ in & AR. & $\varepsilon^{\prime \prime}$ in \\
\hline$=0 \% 038$ & $\cos 40^{\circ} 5$ & $\pm 0^{\prime \prime} 73$ \\
\hline 0,044 & $\cos 40,3$ & 2,12 \\
\hline 0,175 & $\cos 40,3$ & 0,57 \\
\hline 0,170 & cos 39,9 & 2,25 \\
\hline 0,117 & $\cos 39,8$ & 1,77 \\
\hline 0,099 & $\cos 37,8$ & 0,60 \\
\hline 0,090 & ros 37,8 & , $\quad 0,76$ \\
\hline 0,089 & $\cos 37,6$ & 0,98 \\
\hline 0,044 & $\cos 37,5$ & 1,11 \\
\hline 0,010 & $\cos 37,3$ & 0,62 \\
\hline 0,056 & $\cos 36,6$ & 0,91 \\
\hline 0,011 & cos 35,9 & \\
\hline 0,030 & $\cos 34,0$ & 1,14 \\
\hline 0,032 & $\cos 32,4$ & 0,59 \\
\hline 0,112 & $\cos 31,9$ & 1,59 \\
\hline 0,078 & $\operatorname{ros} 31,4$ & 0,91 \\
\hline 0,054 & $\cos 30,9$ & 0,84 \\
\hline 0,020 & $\cos 30,9$ & 0,44 \\
\hline 0,053 & $\cos 28,9$ & 1,32 \\
\hline 0,052 & $\operatorname{ros} 28,1$ & 0,32 \\
\hline 0,065 & $\cos 26,3$ & 0,48 \\
\hline 0,076 & $\cos 25,3$ & 2,37 \\
\hline 0,054 & $\cos 24,1$ & 2,12 \\
\hline 0,086 & $\cos 22,8$ & 0,68 \\
\hline $\pm 0,044$ & $\cos 21,3$ & $\pm 0,65$ \\
\hline
\end{tabular}

Die grössere Genaugkeit, mit weleher sich gegen das Ende der Erscheinung der Comet hetrachten liess, wurde durch den tiefen Stand zuletat wieder aufgehoben. Die folgenden Zahlen lissen dies besser erkennen.

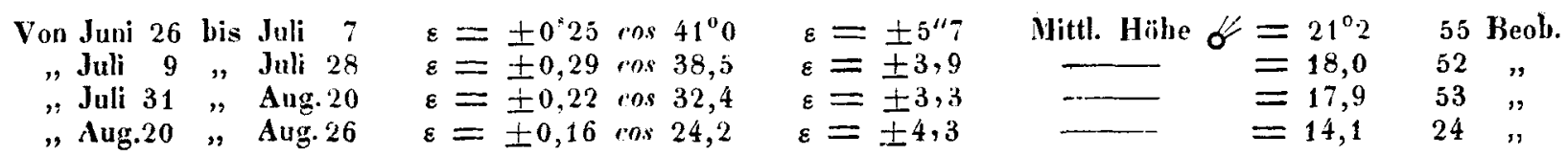

Aus 183 einzelnen Durchgängen finde ich nach einer andern Rechnung für den j fuss. Refractor

$$
\begin{array}{lll}
\varepsilon= \pm 0^{\circ} 240 \cos 35^{\circ} 42 & \varepsilon \pm 4^{\prime \prime} 39 & \text { Mittlere Hihe of } 17^{\circ} 8 \\
\varepsilon^{\prime \prime}= \pm 0,071 \text { cos } 35,42 & \varepsilon^{\prime \prime} \pm 1,31 & \text { für ein Mittel aus je } 5 \text { Durchgängen. }
\end{array}
$$

Für den 3 fuss. Plössl finde ich endlich

$$
\varepsilon= \pm 0^{\circ} 300 \text { ass } 26^{\circ} 8 \quad \varepsilon \pm 3^{\prime \prime} 60 \quad \text { Mittlere Hïhe }=8^{\circ} 9 \text {. }
$$

J. F. Jul. Schmidt,

Olmütz 1853, Sept. 14.

Observator der Sternwarte zil Olmütz.

Beolichtungen, Elementc und Ephemeride des Cometea IV. 1853.

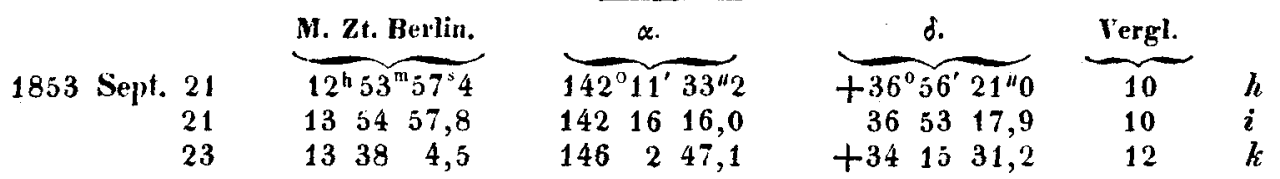

Mittlere Oerter der Vergleichsterne für 1853,0.
h $\quad 141^{\circ} 18^{\prime} 4^{\prime \prime} 6 \quad+37^{\circ} 2^{\prime} 49^{\prime \prime} 9$
B.Z. 407,499 ; Lal. 18757 ; Taylor 4201 .
$\begin{array}{llll}i & 141 & 37 & 46,8 \\ k & 145 & 18 & 54,6\end{array}$
$+365634,7$
$+3417 \quad 3,7$
B. Z. $49 j$; Lal. 19234 , 19235 .

Bei $* h$ ist Taylor doppeltes Gewicht, bei $* k$, Bessel doppeltes Gewicht gegeben.

Fast bei allen Beobachtungen bat Herr Lesser und bei den letaten auch Herr Hoffmann Hülfe geleistet. 